\title{
A Multimodal Discourse Analysis of Second Language Instruction Videos
}

\author{
Jie Zhu \\ Zhejiang Yuexiu University, Shaoxing, China \\ Email: 20131050@zyufl.edu.cn
}

How to cite this paper: Zhu, J. (2021) A Multimodal Discourse Analysis of Second Language Instruction Videos. Open Access Library Journal, 8: e7651.

https://doi.org/10.4236/oalib.1107651

Received: June 16, 2021

Accepted: October 12, 2021

Published: October 15, 2021

Copyright $\odot 2021$ by author(s) and Open Access Library Inc.

This work is licensed under the Creative Commons Attribution International License (CC BY 4.0).

http://creativecommons.org/licenses/by/4.0/

\section{(c) (i) Open Access}

\begin{abstract}
As the internet and education technologies boom, second language teaching has been witnessing profound changes. The second language instruction video is one of the changes mentioned. This paper aims to construct a framework to study the instruction videos based on the Visual Grammar Theory of Social Semiotics and Power Distance theory and then applies this framework to the analysis of prize-winning instruction videos in China, so as to find out the features in narration, representational meaning, interactive meaning, compositional meaning and the ideology behind the videos. The significance of this study is twofold, one is to illustrate that video analysis should be done with reference to cultural theories and an analytical framework should be built according to a specific genre. The other is to discuss how to effectively build the identity of teachers in the digital era, and provide some suggestions for future police discourse practice.
\end{abstract}

\section{Subject Areas}

Language Education

\section{Keywords}

Second Language Instruction Video, Multimodal Discourse, Identity of Second Language Teachers

\section{1. 引言}

信息技术与外语课程教学的深度融合已经成为外语教育发展的趋势, 目 前教学模式和教学方法发生了深刻变化, 短视频内容输出成为重要教学手段, 得到广泛应用。与传统教学中主要依靠文字语言表达意义和交流不同, 短视 频融合文本、图像、声音、动作等多模态符号资源辅助教学, 不仅具有教育 
性同时伴有娱乐性。但是大部分一线教师没有受过教学视频制作的专业培训, 不清楚如何运用听觉、视觉、触觉等多种感觉, 通过语言、图像、声音、动 作等多种手段和符号资源进行交际, 可能导致教学效果欠佳。因此理清多模 态符号资源在教学中的使用情况, 特别是图像资源的使用情况非常重要 [1]。 为此, 国内许多学者展开大量的理论探索和实证研究, 包括外语教学、广告、 宣传片、海报、画作等方面。其中语言教学领域的多模态研究涉及: 外语教 学模式研究 [1] [2], 多元识读能力培养研究 [3] [4], 儿童言语行为研究 [5], 多 模态听说教学 [6], 多模态教材研究 [7] [8], 多模态 PPT 演示教学研究 [9] [10]。

尽管多模态研究的理论和应用研究日益丰富, 但是对于外语微课的多模 态特征和功能的研究还不多。微课是以阐述某一知识点为目标, 以短小精炼 的视频为表现形式, 以网络为传播途径, 以学习或教学应用为目的的教学视 频[11], 因此微课属于拥有网络话语特征的多模态教学话语。近年来, 许多学 者意识到单从语言的角度来研究课堂教学话语是有缺陷的, 必须把语言和其 他模态组合在一起来探讨教学话语的意义构建过程。他们从新的的角度探究 课堂教学话语, 比如教师话语与手势语的符际关系[12]。慢慢也有教师注意到 外语微课的研究 [13] [14]但是他们都是从应用语言学的角度分析优秀微课的 特点和问题并给出建议。以上研究从教学话语的符际关系、传播效果方面论 述多模态教学话语, 却忽视了话语不仅是交际工具和知识载体, 也是一种权 力工具。微课作为教学话语的新形式, 是一种社会实践, 是教师在特定语境 中运用语言及其他手段达到教学目标的一种交际活动。吴婷[15]整合视觉语法 和图文关系理论分析微课中的图文关系, 但是她只对 2 个微课视频作对比。 刘立香和吴琦[16]只讨论了外语微课中的模态使用情况, 没用对外语微课采取 哪些具体的话语策略或符号建构教师身份、构建意义、师生互动等问题并没 有展开深入的探讨。

鉴于此, 本文以第六届全国高校外语微课大赛获得一等奖的 22 个视频为 分析对象, 根据宣传片的语类特征, 结合社会符号学和传播学理论进行分析 和讨论, 回答三个研究问题: 1) 优秀外语微课有什么语类特征, 即通常包括 哪些语步? 2) 它们使用哪些符号资源, 建立教师与微课学习者之间的互动关 系？3）外语微课设计体现了什么教学理念?

\section{2. 理论背景}

1996 年 Kress \& Van Leeuven [17]出版《阅读图像——视觉设计语法》 (Reading Images: the Grammar of Visual Design), 创立视觉语法, 此后多模态 话语分析开始蓬勃发展。多模态话语分析的本质是探索语言与其他符号模态 如何共同作用从而实现人类交际活动, 但是研究对象和研究路径不尽相同, 体现明显的跨学科性和跨学科性。目前主要有九个研究路径: 系统功能语言 学多模态话语分析、社会符号学、多模态认知分析、多模态互动分析、会话 分析、地理符号学、多模态民族志、多模态语料库分析以及多模态感知分析 [18]。本文的分析对象是教学短视频, 涉及图像、语言、声音等多种符号特征, 主要借鉴社会符号学相关理论。社会符号学, 作为批评话语分析的一个流派, 强调符号意义的社会性, 认为社会政治文化环境以及符号使用者个人的兴趣、 
目的会影响符号资源的选择, 因此意义是选择的结果。社会符号学关注符号 使用的社会效应和意识形态效果，旨在揭示多模态语篇在设计、生产和传播 过程中权力和意识形态的作用。尽管社会符号学将语言符号之外的其他符号 系统(例如: 图像、数字、色彩和面部表情等)都纳入研究范围, 但在众多符 号模态中, 图像和文字仍然是最主要的信息载体和研究对象 [17]。

视觉语法[17]是社会符号学的主要理论, 借用 Halliday[19]的语言三大功 能假说, 提出视觉图像分析的三个维度: 图像的再现意义、互动意义和构图 意义, 分别对应语言三大功能的概念功能、人际功能和语篇功能。首先, 图 像的再现意义分为图像叙事关系和元素结构, 表示图像中人、事、地之间的 关系或概念; 其次, 互动意义旨在揭示图像提供者与观看者之间的交流方式 与认知态度, 通过距离、接触、视角和情态手段把握; 最后, 构图意义可以 由信息值、显著性和框架手段实现[17]。

\section{3. 外语微课多模态话语分析框架}

多模态话语分析的理论视角和分析路径众多, 为语篇分析提供了一个工 具箱, 具体使用哪些工具, 取决于分析对象的语类。也就是说, 要针对不同 的语类特点来选择分析的意义要素, 构建适合特定语类的分析框架 [18]。本文 的分析对象是 21 个第六届全国高校外语微课比赛一等奖视频, 视频围绕外语 学科某一主题和知识点, 有秩序、有重点地策划、拍摄、剪辑成片并以网络 为传播途径, 目的是让目标观众接受某种信息。本文讨论重点是这 21 个外语 微课视频主题是否有相似的叙事特征, 即教学步骤, 除语言以外的其他符号 资源使用情况。说话人与听话人之间的角色关系、符号资源的组织方式, 即 外语微课中教师设计和观众建立什么样的关系, 如何利用符号资源完成信息 传播。因此本文主要借鉴体裁分析及社会符号学在再现、互动和构图意义方 面的论述构建分析框架, 如图 1 所示。

\section{1. 体裁特征}

体裁是具有共同交际目的, 可辨认的、内部结构特征鲜明的、高度约定 俗成的一组交际事件 [20]。在体裁分析框架中, 语步是执行同一个交际功能的 语篇单元, 具有较为灵活的语言实现形式, 既可以是一句话或一段话, 也可 以是由几段话组成的篇章[21]。吴婷[16]分析 2014 年第一届外语微课大赛两 个一等奖作品总结出外语微课的三个语步特征: 引入 - 展开 - 总结。本文在吴 婷分析的基础上将微课视频分为七个语步, 其中部分是必要语步, 部分是可

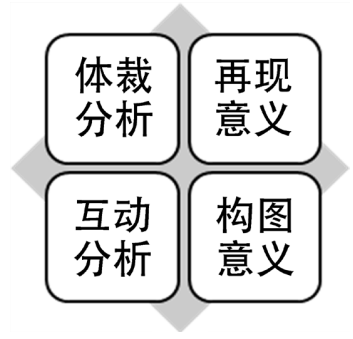

图 1. 微课多模态话语分析框架 
选语步, 如表 1 所示。

\section{2. 图像再现意义}

Kress \& van Leeuwen [17]把图像区分为叙事类和概念类, 叙事图像表征 动作和事件展开、过程变化和空间转变, 带有矢量, 而概念图像表示图像的 结构、类别和意义, 无矢量。本文将微课中的提取图像, 如主讲教师、主讲 教师意外的其他人物、动物、事物、卡通形象等, 根据 Wilson 和 Landon-Hays [22]对图像编码风格取向, 将图像分为三大类: 示意图风格取向 schematic orientation、自然风格取向 naturalistic orientation (比如真人、真物照片)、艺 术风格取向 artistic orientation (比如画作、卡通形象)。

\section{3. 图像互动意义}

互动意义主要从镜头距离、目光接触、拍摄角度和情态四种方式来实现。 Wilson 和 Landon-Hays [22] 认为图像编码风格取向与情态关系密切, 反映图 像对现实世界表征的真实度或者可信度。示意图风格取向的图像保留事物的 普遍特征, 排除其他无关干扰, 是可信度最高的一种图像, 常常用在科技话 语中。自然风格取向的图像, 比如照片, 与我们生活的真实世界非常接近的, 因此意味着客观不带偏见, 也具有非常高的真实性。艺术风格取向的图像和 客观世界的所见不同, 不是客观世界直接的反映, 但是可以从情绪情感上召 唤观众, 从而影响变观众。Painter et al. [23] 认为图像表征的写实、抽象等风 格不仅是情态问题, 而是作者设定读者与图像人物情感关系的策略。因此, 教师真人图像属于个人风格(personalized style), 最易召唤观众情感; 教师卡 通图像属于类化风格 (generic style)较之教师真人图像, 召唤情感能力较弱。 本文在 Kress \& Van Leeuwen [17]互动分析方法的基础上加上 Wilson \& Landon-Hays [22]图像风格取向的分析和 Painter [23]的氛围系统分析形成图像互 动意义实现方式分析(如表 2)。

\section{4. 构图意义}

Painter [23] 将图像和语言的关系分为融合与互补两种: 融合关系即语言 表 1. 微课视频语步分析

\begin{tabular}{ccl}
\hline 序号 & 语步 & \multicolumn{1}{c}{ 作用 } \\
\hline 1 & 微课标题 & 针定话题 \\
2 & 导入 & 介绍知识点或引发学生的学习兴趣 \\
3 & 学习目标 & 介绍学习学生能够学到的知识、发展的能力 \\
4 & 介绍知识点 & 给出知识点的定义 \\
5 & 详解 & 解释说明定义 \\
6 & 举例 & 以样例进一步解释知识点 \\
7 & 总结 & 回顾所学知识点 \\
8 & 作业 & 课后巩固练习知识点 \\
\hline
\end{tabular}


表 2. 图像互动意义的实现方式

\begin{tabular}{cc}
\hline 图像行为 & 实现方式 \\
\hline 索取 & 凝视观众 \\
提供 & 没有凝视 \\
亲密的/个人的关系 & 近景镜头(人物的胸部以上) \\
社会关系 & 中景镜头(人物的腰部以上) \\
非个人的 & 远景镜头(表示人物与环境的关系) \\
观者的权力 & 俯拍角度 \\
平等的关系 & 平角角度 \\
图像参与者的权力 & 仰拍的角度 \\
高真实性 & 示意图风格取向 \\
高真实性 & 自然风格取向 \\
强情感 & 艺术风格取向 \\
强情感 & 真人教师 \\
弱情感 & 卡通形象教师 \\
活力 & 鲜亮色彩 \\
克制 & 灰暗色彩 \\
温馨、舒适 & 暖色调 \\
冷漠、疏远 & 冷色调 \\
熟悉 & 色彩丰富 \\
陌生 & 色彩单一 \\
\hline
\end{tabular}

与图像合为一体, 没有明显界限; 互补关系即语言与图像分别占一部分空间, 相互有明显界限。融合关系中有两种子关系: 一、话语投射, 主要通过语泡 实现, 也可以没用话语泡; 二、扩展指图像与文字具有各自的意义, 两者相 互阐释、补充、或增强。互补关系中，可从对称性、重要性与位置特征三个 角度考察其互补关系。

\section{4. 外语微课教学话语多模态分析}

\section{1. 内容描写和语类特征分析}

第六届全国高校外语微课大赛一等奖获奖微课共 21 个, 涉及语种包括英 语、日语、法语。根据主题可分为四大类微课: 语言知识类 $(10$ 个)、文化介 绍类 $(5$ 个)、专门用途英语类 ( 3 个)、演讲技巧类 $(3$ 个)。通过手工标记统计考 察外语微课的语步结构, 结果如表 3 所示。尽管主题不同, 外语微课的结构 和语步相似, 具有教学语篇特征, 21 个微课都包含微课标题、导入、知识点 介绍、详解、示例、总结六个语步, 2 个视频没有包含学习目标和 11 个视频 没有包含知识点相关练习题。如果微课是一个独立教学视频, 将学习目标和 练习设计在视频中, 可以使学习者在学习前明确学习目标, 在学习后检验学 习目标达成情况。如果微课是系列课程之一, 另行安排学习目标与练习, 也 
是可行的。

\section{2. 图像再现意义分析}

在 22 个微课视频中有 1 个视频完全采用动画形式制作, 1 个视频中除教 师出境部分其余采用动画形式制作, 15 个视频采用视频链接加 PPT 动画加讲 解, 剩余 5 个视频采用 PPT 动画加讲解制作。除去视频链接, 通过对微课 PPT 动画中图像的仔细观察和分类，表示三种不同类型的图片运用情况统计如下 表 4, 艺术取向图像的偏好非常明显, 65\%的图像属于艺术取向。说明外语教 师在设计微课时, 并不十分在意图像传达信息的真实性和可靠性, 他们像作 家创作文学作品一样, 试图展示与真实世界相似但又不是完全一致的图像。

另外, 人物的表征可以通过衣着特征代表人物身份, 形成转喻关系[23]。 在 21 个有主讲教师出境的视频中(包以卡通人物形象), 所有教师都穿着职业 装(如图 2、图 3), 凸显正式的社交场景。

\section{表 3. 微课视频语步分析}

\begin{tabular}{cccc}
\hline 序号 & 语步 & 作用 & 频率 \\
\hline 1 & 微课标题 & 针定话题 & $21(100 \%)$ \\
2 & 导入 & 介绍知识点或引发学生的学习兴趣 & $21(100 \%)$ \\
3 & 学习目标 & 指出学生学习视频后要达到的知识、能力目标 & $2(9 \%)$ \\
4 & 介绍知识点 & 介绍知识点的信息 & $21(100 \%)$ \\
5 & 详解 & 解释说明知识点 & $21(100 \%)$ \\
6 & 示例 & 诉诸具体例子解释复杂概念 & $21(100 \%)$ \\
7 & 练习 & 与知识点相关练习题 & $11(50 \%)$ \\
8 & 总结 & 回顾所学知识点 & $21(100 \%)$ \\
9 & 课后作业 & 课后巩固练习知识点 & $17(77 \%)$ \\
\hline
\end{tabular}

表 4. 外语微课视频图像类型统计表

\begin{tabular}{cccc}
\hline 图像类型 & 示意图取向 & 自然取向 & 艺术取向 \\
\hline 数量 & 4 & 170 & 318
\end{tabular}

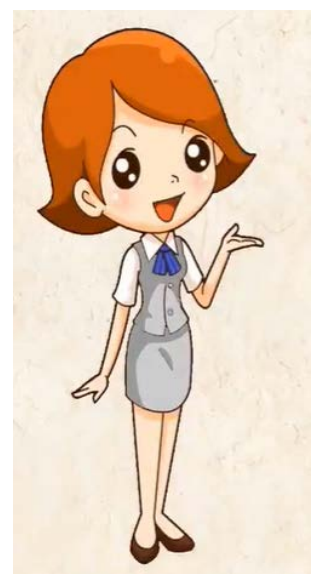

图 2. 卡通主讲教师形象 


\section{3. 互动意义分析}

在 22 个微课视频中, 主讲教师出境的视频有 19 个, 主讲未出境的只有 3 个。外语微课的互动意义从主讲教师的镜头距离、目光接触、拍摄角度和 图像表征的情态四种方式来分析。拍摄出境教师时的角度、目光接触、距离 统计入表 5。首先所有镜头都是平角, 摄像机镜头与被拍摄对象即教师处于 水平的位置, 这是常用的拍摄角度, 符合人们观察世界的角度, 观众和图像 参与者是平等的关系。其次所有出境教师都与观众有目光交流, 即图像参与 者和观众有目光交流, 图像先观看者发出邀请, 索取观众的共鸣, 是 “索取” 图像(100\%)。第三个微课中大部分教师镜头(85\%)运用远景, 只有 $15 \%$ 的教师 镜头属于中镜头。图像参与者和观看者之间的关系越亲密, 镜头与参与者距 离越小。远镜头主要用于建立非个人的社会关系; 中镜头揭示一般的社会关 系。除教师镜头以外的其他人物图像 $90 \%$ 是远镜头, 与观众没有对视。

Wilson 和 Landon-Hays [22] 认为图像编码风格取向与情态关系密切。微 课中大量使用卡通图像 (65\%), 说明外语类教学短视频并不通过理性展现现实 世界, 他们更倾向于通过图像达到召唤学习者的学习兴趣的目的。

图像的颜色是揭示情态的重要特征 [23], 整体来说外语微课的色度偏低, 色调偏浅, 颜色单一, 这与外语微课以语言教学为目标有关(表 6)。在标题、

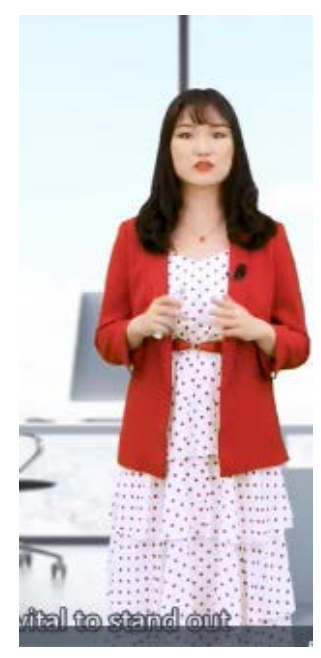

图 3. 真人主讲教师形象

表 5. 外语微课镜头互动意义统计

\begin{tabular}{cccccc}
\hline 主讲人图像 & 平角 & 接触 & 近镜 & 中镜 & 远镜 \\
\hline 数量 & $20(100 \%)$ & $20(100 \%)$ & $0(0 \%)$ & $3(15 \%)$ & $17(85 \%)$ \\
\hline
\end{tabular}

表 6. 外语微课标题、导入部分颜色互动意义统计

\begin{tabular}{cccc}
\hline 标题图像颜色 & 高色度 & 暖色调 & 颜色丰富 \\
数量 & $36 \%$ & $40 \%$ & $35 \%$ \\
导入图像颜色 & 高色度 & 暖色调 & 颜色丰富 \\
数量 & $30 \%$ & $50 \%$ & $40 \%$ \\
\hline
\end{tabular}


导入部分有一些微课尝试使用高饱和色度、暖色调而且颜色丰富, 以此拉近 与观众的距离, 唤起观众的共鸣。在知识讲解、示例部分, 通常以语言模态 为主, 背景色倾向选取饱和度低的颜色、冷色调, 且颜色较单一的。

在互动策略方面, 外语微课以视觉(画面)和语言两种符号模态为主, 21 个视频(100\%)都借助动画效果形式吸引学习者注意力。同时, 有 7 个微课(33\%) 借助声音吸引学习者注意力。其中有 2 个微课在教师讲解过程中设置背景音 乐, 一个是日语词汇教教学视频, 另一个是中国文化教学视频。两首背景音 乐都节奏欢快, 给人轻松愉悦的感觉。有 5 个微课(分别 3 个英语知识微课、 1 个演讲微课、 1 个文化微课) 设置特殊音效, 比如计时器声音、鼓掌声、专 场音效等, 活跃气氛, 以期增加学生的学习兴趣。

\section{4. 构图意义分析}

获奖外语微课中, 没有出现语言与图像毫无关联的情况, 说明视频制作 教师具备一定的多元识读能力。微课中的语言与图像主要是互补关系, 图像 和语言有明显的空间分隔(如图 4)。外语微课中, 对称是最常见的互补关系实 现方式(如图 4), 且以文字为主, 文字起到针定意义的作用, 文字通常有边框, 与图像隔离。所有微课中, 只有 5 个微课中图文出现融合关系, 它们以话语 泡的形式显示话语投射。

\section{5. 分析与讨论}

在上述分析结果的基础上, 我们对外语微课中多模态符号使用情况、受 众地位分别从外语教学和文化角度加以分析讨论。

国内许多学者的研究成果表明, 多模态教学在激发学生学习兴趣, 增强 学生多元识读意识和技能, 提升学生自主学习能力等方面都起到积极影响的 作用 [1] [3] [9]。从第六届全国外语微课大赛的一等奖作品中可以发现外语教 师与时俱进, 信息技术的意识和能力已经得到非常大的提高。教师在借助媒 体技术教授外语的过程中, 教师扮演多重角色, 外语专家、教学专家、视频

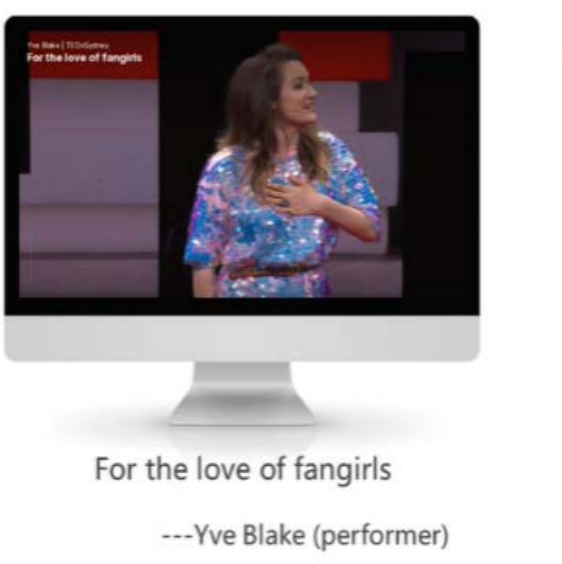

\section{$\star$ Transition}

\section{A fangirl's story}

of being infatuated with Harry Styles, an English pop singer and actor,

图 4. 图文对称 
创造者。教师通过微课传授知识点, 解放课堂时间, 从而将实体课堂变为交 流、研究、反思、合作的空间而不是灌输知识的场地。

从外语微课的符号使用分析可以发现, 教师虽然在积极借助图像和声音 模态召唤学生的学习兴趣, 但在情感交流方面仍然有不足之处。在镜头距离 的选择、视频主题色彩的选择上, 大部分教师仍然保持着实体课堂上的距离 感, 教师的权威角色并没有淡化, 这不利于学生平等、深度参与课堂意义构 建。教师难以摆脱权威身份的原因与我国的集体主义文化有密切关系。根据 Hofstede [24]提出的权力距离理论, 即在社会中分配到较少权力的那部分社会 成员对权力分配不平等状况的承受度。Hofstede 按照权力距离高低将文化分 为高权力距离文化与低权力距离文化, 研究显示, 世界平均权力距离指数水 平为 55 , 英国、美国、加拿大、澳大利亚等西方国家的权力指数低于 55 , 属 于低权力距离文化国家; 而中国的权力指数高达 80 , 属于高权力距离文化国 家[25]。Hofstede 认为集体主义倾向越高的文化, 权力距离越高, 也就是说, 在越是推崇集体主义文化的社会, 相较于其他类型社会文化, 社会成员对权 力分配不平等状况的接受度越高。因此中国社会推崇重视权力、权威与身份 差异, 比如师道尊严、尊师重教, 教师与学生之间有明显的权力距离, 交往 时约束较多。权力距离直接影响外语微课教学中教师身份的建构以及微课互 动中的表现。

\section{6. 结束语}

本文运用社会符号学的视觉语法理论, 借鉴权力距离理论, 建构外语微 课多模态话语的分析框架, 运用这个框架分析了 22 个外语微课获奖作品, 揭 示了外语微课的语步特征、概念意义、互动意义以及构图意义, 并就微课所 反映的受众地位进行了探讨。本文的研究意义在于, 一是尝试将微课视频分 析结合社会符号理论、权利距离理论和观点, 针对微课这一语类特征建立分 析框架; 二是尝试探讨教师在信息化社会中身份建构的有效途径, 为微课教 学话语的研究贡献微薄之力, 在进一步深化大学英语教学改革的新形势下, 为今后如何组织教学内容以及选择什么样的模态来传达特定的教学内容; 如 何调用和协同各种模态从而有效表达教学内容; 如何使教学内容能够兼具知 识性、实用性及趣味性提供一些借鉴。

\section{基金项目}

本文为浙江越秀外国语学院校级科研项目一一外语微课超文化身份建构 多模态研究阶段性成果。

\section{Conflicts of Interest}

The author declares no conflicts of interest.

\section{References}

[1] 张德禄. 多模态话语理论与媒体技术在外语教学中的应用[J]. 外语教学, 2009, 30(4): 15-20. 
[2] 李颖. 虚拟现实(VR)与外语教学模态再建研究[J]. 外语电化教学, 2020(1): 24-30.

[3] 邢春燕, 冯德正. 多媒体环境下大学英语课堂教学设计与评估: 多元读写理论视 角[J]. 山东外语教学, 2019, 40(3): 41-51.

[4] 张德禄, 刘睿. 中国大学生外语多元读写能力评估框架探索 [J]. 当代外语研究, 2015(9): 26-31, 32.

[5] 黄立鹤, 张德禄. 多核并行架构: 多模态研究的范式、路径及领域问题之辨 [J]. 外 语教学, 2019, 40(1): 21-26.

[6] 陈新. 基于多模态理论框架的汉语视听说教学模式设计与研究[J]. 云南大学学报 (自然科学版), 2020, 42(s1): 116-122.

[7] 冯德正. 英语教学中的人文道德教育: 正面价值观的多模态语篇建构[J]. 外语界, 2015(5): 27-34.

[8] 张德禄, 张淑杰. 多模态性外语教材编写原则探索[J]. 外语界, 2010(5): 26-33.

[9] 胡壮麟, 董佳. 意义的多模态构建一一对一次 PPT 演示竞赛的语篇分析[J]. 外语 电化教学, 2006(3): 3-12.

[10] 张征. 多模态 PPT 演示教学与学生学习绩效的相关性研究 [J]. 中国外语, 2010, 7(3): 54-58.

[11] 胡铁生, 黄明燕, 李民. 我国微课发展的三个阶段及其启示 [J]. 远程教育杂志, 2013(4): 36-42.

[12] 王珊, 刘峻宇. 国际汉语词汇教学中的多模态话语分析 [J]. 汉语学习, 2020(6): $85-96$.

[13] 张吉. 二语教学微课设计存在的问题及优化策略 [J]. 西安外国大学学报, 2021, 29(1): 69-72.

[14] 马武林, 何静静. 优秀外语微课特征分析一一以中国外语微课大赛一等奖作品为 例[J]. 外语电化教学, 2017(2): 22-26.

[15] 吴婷. 外语微课的多模态话语符际间性研究一一以两则微课大赛视频为例 [J]. 外 语教学理论与实践, 2017(1): 72-77.

[16] 刘立香, 吴琦. 优质英语慕课的多模态教学特征及前景化手段[J]. 集美大学学报 (教育科学版), 2019, 20(6): 59-66.

[17] Kress, G. and van Leeuwen, T. (1996) Reading Images: The Grammar of Visual Design. Routledge, London.

[18] 潘艳艳. 多模态视阈下的国家安全话语分析一一以中美警察形象宣传片的对比 分析为例 [J]. 外国语文, 2019, 35(1): 78-87.

[19] Halliday, M.A.K. (1994) An Introduction to Functional Grammar. 2nd Edition, Arnold, London.

[20] Bhatia, V.K. (1993) Analyzing Genre: Language Use in Professional Settings. Longman, London.

[21] Bhatia, V.K. (2004) Worlds of Written Discourse. Continuum, London.

[22] Wilson, A. and Landon-Hays, M. (2016) A social semiotic analysis of instructional images across academic disciplines. Visual Communication, 15, 3-31. https://doi.org/10.1177\%2F1470357215609213

[23] Painter, C., Martin, J.R. and Unsworth, L. (2013) Reading Visual Narratives: Image Analysis of Children's Picture Books. Equinox, London.

[24] Hofstede, G. (1991) Cultures and Organizations: Software of the Mind. McGraw-Hill, London.

[25] 间文培. 全球化语境下的中西文化及语言对比[M]. 北京: 科学出版社, 2007. 


\section{Appendix (Abstract and Keywords in Chinese) 外语微课教学话语的社会符号研究}

摘要: 随着互联网的发展和教育技术的进步, 外语教学模式发生了深刻变化, 短视频内容输出成为重要教学手段, 得到广泛应用。本文运用社会符号学的 视觉语法理论同时借鉴权力距离理论, 建构外语微课多模态话语的分析框架, 运用这个框架分析了 22 个第六届全国外语微课一等奖获奖作品, 揭示了外语 微课的语步特征、概念意义、互动意义以及构图意义, 并就微课所反映的受 众地位进行了探讨。本文的研究意义在于, 一是尝试将微课视频分析结合社 会符号理论、权利距离理论和观点, 针对微课这一语类特征建立分析框架; 二是尝试探讨教师在信息化社会中身份建构的有效途径, 为微课教学话语的 研究贡献微薄之力。

关键词：外语微课视频，多模态话语，教师身份 